\title{
Brittle Fracture and Spinnability of Viscous Materials
}

\section{$\operatorname{AUTHOR}(\mathrm{S}):$}

Gotoh, Rempei; Aida, Hiroshi; Hayashi, Soichi; Hirai, Nishio

\section{CITATION:}

Gotoh, Rempei ... [et al]. Brittle Fracture and Spinnability of Viscous Materials. Bulletin of the Institute for Chemical Research, Kyoto University 1957, 35(1-2): 26-35

\section{ISSUE DATE:}

1957-10-31

URL:

http://hdl.handle.net/2433/75580

RIGHT: 


\title{
Brittle Fracture and Spinnability of Viscous Materials*
}

\author{
Rempei Gotoh, Hiroshi Aida, Soichi Hayasmr and Nishio Hrra ${ }^{* *}$ \\ (Gotō Laboratory) \\ Received July 1, 1957
}

Types of mechanical fracture or break-down of viscous materials such as rosin, asphalts and plasticized high polymers were examined at various rates of extension. It was found that the critical velocity for brittle fracture and for dropping break-down increased exponentially with increasing temperature. Creeping or spinnable extension was observed between these two limiting conditions.

For the aqueous solutions of glycerine, cane sugar etc., spinnability was examined by the pulling up method. It was suggested that spinnability at a constant rate of extension $(v)$ depends largely upon viscosity $(\eta)$ and surface tension $(\gamma)$ as well as temperature. Assuming that the apparent relaxation time can be given by a balance between viscosity and surface tension, the spinning condition was derived as follows :

$$
\eta \geq \gamma / v \text {. }
$$

The spinning temperature expected from the above relation showed a good agreement with the experimental results.

\section{INTRODUCTION}

Under mechanical stress in tension, solid or plastic materials show elastic elongation or plastic flow and separate into two pieces under a certain limiting condition; the latter case is fracture or brealk-down. It has been known that most materials show several types of fracture in accordance with the rate of deformation as well as with the temperature as shown schematically in Fig. 1. Brittle fracture is an extreme case. In this case, the separation is normal to

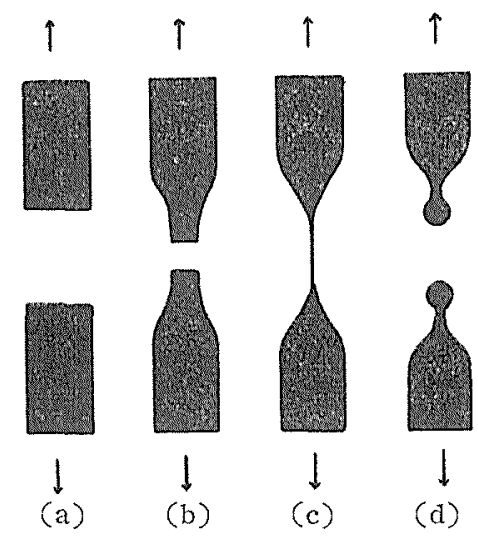

Fig. 1. Types of fracture.

* Published in Japanese in the Journal of the Japan Society for Testing Materials, 6 245 (1957).

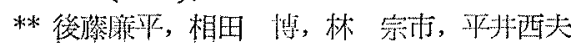


that surface of separation and is followed by almost no deformation of the whole body as shown in Fig. 1 (a). The fracture of this type can be seen above a critical velocity of extension at a definite temperature or below a critical temperature, i.e. the brittle temperature, at a low rate of extension. This critical velocity of extension may be called "the brittle velocity" in contrast to the brittle temperature. At higher temperatures, those materials liquefy and make drops on separation as shown in Fig. 1(d). This is another extreme case of fracture. Between these extreme case, we can find the creeping or spinning condition* with respect to the rate of extension and the temperature (cf. Fig. $1 \mathrm{c}$ ).

In spite of numerous investigations reported, ${ }^{1{ }^{1 a-e}},{ }^{2) a-a}$ the exact natures of fracture and spinning as well as the relation between them have not been established yet. The present authors intended to investigate the critical conditions of brittle fracture and spinning of various materials such as rosin dissolved in olive oil, asphalts, plasticized high polymers and some aqueous solutions, which are highly viscous at ordinary condition, and to discuss the relation between them.

\section{EXPERIMENTAL}

Materials. Rosin for industrial use was dissolved in olive oil at various concentrations. Straight and blown asphalts used were samples from the Nippon Oil Co. The other meterials used were commercially available ones.

Procedure. (1) Brittle Velocity: The brittle velocity was measured with the apparatus shown diagrammatically in Fig. 2. The dum-bell type space in a brass block was filled with the plastic or highly viscous specimens to be tested. The block consisted of four separable parts, and the cylindrical part of the specimen was $6 \mathrm{~mm}$ in diameter and was $5 \mathrm{~mm}$ long. While the right half of the block was fixed, the other half could be pulled by a rack and pinion with a constant velocity. The pulling velocity could be varied discontinuously between 0.06 and $230 \mathrm{~cm}$ per sec. The specimen was placed in a wooden air thermostat regulated between the room temperature and about $100^{\circ} \mathrm{C}$. The pulling

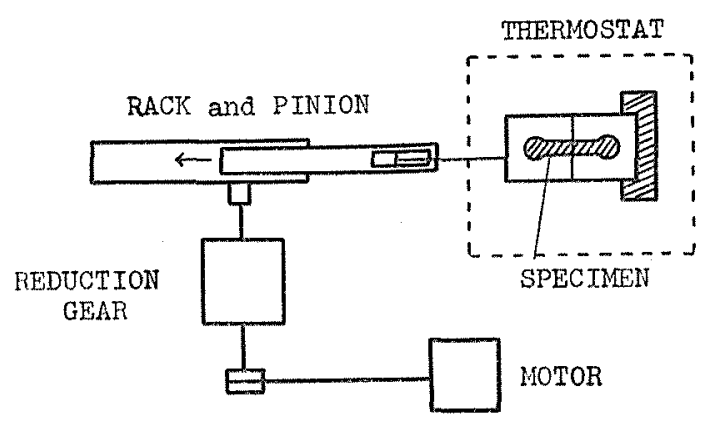

Fig. 2. Extension apparatus.

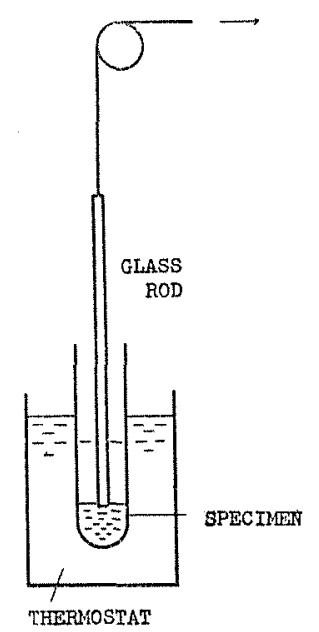

Fig. 3. Spinning apparatus.

\footnotetext{
* Spinning may be considered a special case of creeping in tension, followed by formation of a fine thread.
} 


\section{Rinpei GOTOH et al.}

force given to the fixed part through the specimen was measured with a spring gauge equipped with a optical lever and recorded on a rotating film.

(2) Spinnability: Spinnability was observed with the apparatus shown in Fig. 3. When a glass rod dipped into a viscous material to a definite depth, about $1 \mathrm{~mm}$, was pulled up vertically at a certain constant velocity, the material showed brittle fracture above a certain critical velocity, $V_{n}$, but below $V_{B}$, the material creeped or became spinnable. $V_{B}$ is the brittle velocity. At higher temperatures and a definite velocity of pulling, $V_{s}$, the material becomes less viscous and make drops without spinning. The critical velocity $V_{S}$ may be called the spinning velocity or the dropping velocity.

\section{RESULTS}

(1) Process of Extension. The change in the tensile force during extension was recorded on a rotating film. Fig. 4 shows an example of the results obtained

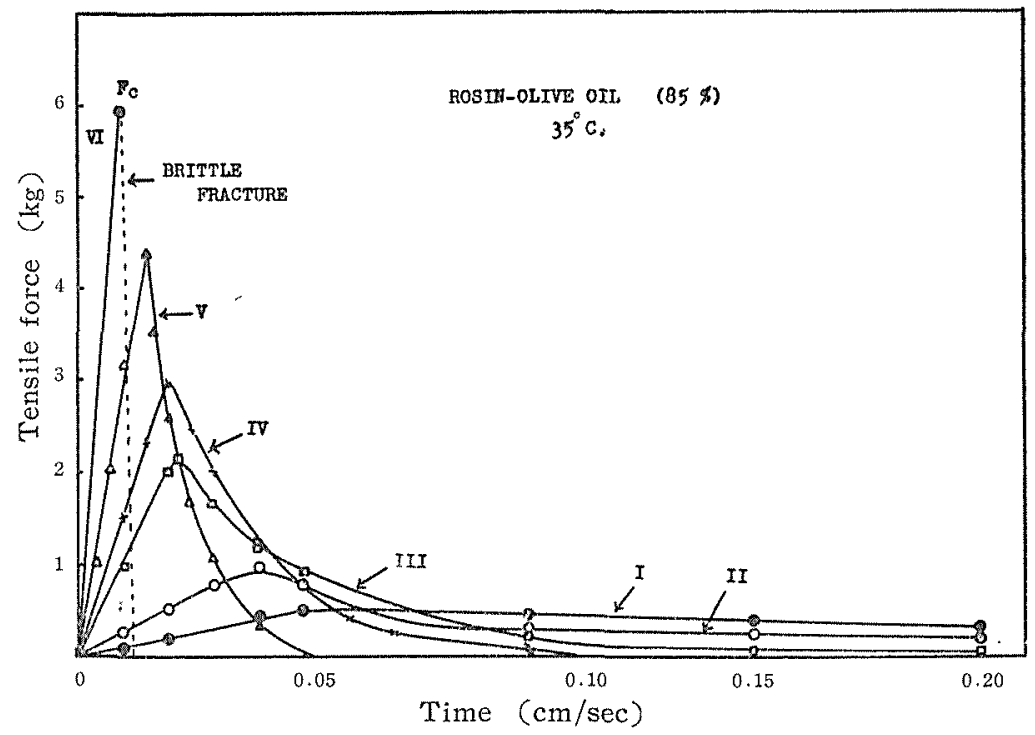

Fig. 4. Change in tensile force during extension of rosin-olive oil.

Rate of extension (cm/sec): I: $0.6 ; \mathrm{II}: 2.5 ;$ III : $6.0 ;$ IV $: 10.0 ; \mathrm{V}: 14.4 ; \mathrm{VI}: 20.4$.

for rosin dissolved in olive oil. In a relatively slow extension, the tensile force reached a maximum and then decreased, being followed by necking or spinning. As the rate of extension was increased, the maximum point came sooner and became higher. When the maximum tensile force reached a definite value, $F_{c}$, no more extension was observed and brittle fracture occurred as shown by a dotted line in Fig. 4. $F_{c}$ may be called the brittle tensile force or the brittle strength. The relation between the rate of extension and the maximum tensile force is shown in Fig. 5. The brittle velocity, $V_{n}$, increased exponentially with increasing temperature and could be expressed approximately by the following relation,

$$
V_{B}=A e^{-B^{\prime} / T}
$$

where $A$ and $B$ are empirical constants characteristic of each specimen. 
Brittle Fracture and Spinnabiiity of Viscous Materials

Table 1, Rosin-olive oil.

\begin{tabular}{ccc}
\hline Conc. of Rosin $\%$ & $A$ & $B$ \\
75 & 63 & $18 \times 10^{3}$ \\
80 & 41 & $12 \mathrm{l}$ \\
85 & 30 & $9 \mathrm{l}$ \\
90 & 21 & $7 \mathrm{~J}$
\end{tabular}

Table 2. Asphalts.

\begin{tabular}{clll}
\hline \multicolumn{1}{c}{ Asphalts } & $A$ & $B$ \\
\hline Straight & & 46 & $13 \times 10^{3}$ \\
Blown & A1 & 40 & 12 "I \\
" & H5 & 40 & 12 " \\
" & Higashiyama & 39 & 12 " \\
" & A5 & 31 & 10 " \\
Semi-blown & 45 &
\end{tabular}

Tables 1 and 2 show the numerical values of those constants for rosin-olive oil and asphalts. While both $A$ and $B$ of rosin-olive oil showed a consiclerable change in accordance with their concentration, the effect of blowing in asphalts could be seen rather in the change of $A$, and the values of $B$ remained almost constant.

Viscosities, $\gamma$, of the specimens were measured with the concentric cylinder method at various temperatures and were shown to be almost Newtonian, though they increased slightly under a very low rate of strain. The viscous resistance at the brittle velocity was defined by ${ }_{\eta} V_{B} / l_{0}, l_{0}$ being the effective length of the specimen. As shown in Table 3, the numerical values of this viscous resistance were nearly of the same order of magnitude with the brittle strength, $F_{c}$.

Table 3. Brittle strength and viscous force of rosin-olive oil.

\begin{tabular}{|c|c|c|c|}
\hline Conc. \% & Temperature, ${ }^{\circ} \mathrm{C}$ & $\begin{array}{c}F_{c} \\
\left(\text { dyne } / \mathrm{cm}^{2}\right)\end{array}$ & $\begin{array}{c}\eta V_{n} / l_{0} \\
\left(\text { dyne } / \mathrm{cm}^{2}\right)\end{array}$ \\
\hline \multirow{5}{*}{85} & 20 & $0.9 \times 10^{7}$ & $1.8 \times 10^{7}$ \\
\hline & 25 & - & $1.6 \%$ \\
\hline & 30 & $1.1 \%$ & $1.4 \mathrm{~N}$ \\
\hline & 35 & $1.0 "$ & 2.5 \\
\hline & 40 & $1.8 "$ & 3.3 \\
\hline \multirow{4}{*}{ · 90} & 30 & $0.9 \times 10^{7}$ & $4.9 \times 10^{7}$ \\
\hline & 35 & $1.2 \%$ & $3.0 "$ \\
\hline & 40 & $1.9 \mathrm{~J}$ & 3.3 \\
\hline & 45 & 2.9 & 5.4 I \\
\hline
\end{tabular}

These results were expressed approximately by the relation

$$
\eta_{1} V_{B}{ }^{\alpha} / l_{0}=\mathrm{const}=F_{e}
$$

where $\alpha$ is a certain constant nearly equal to 1 . This relation suggests that the brittle strength of the viscous material corresponds to the viscous force at the 


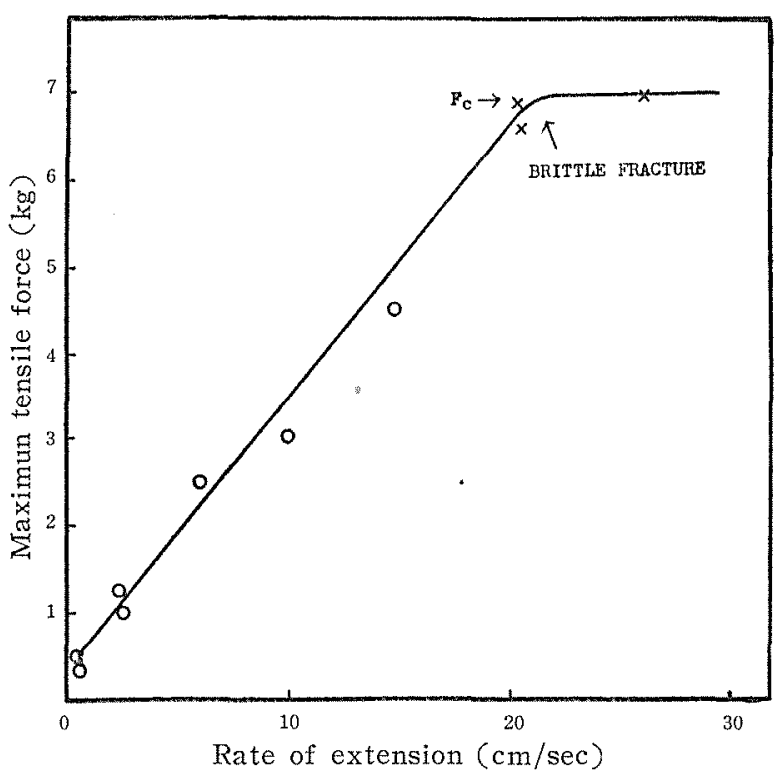

Fig. 5. Maximum tensile force of rosin-olive oil.

moment of the brittle fracture.* It was assumed in the above consideration that the viscosity was independent of the rate of strain on account of the fact that the resistance force increased almost linearly with increasing rate of strain unti1 it reached the brittle strength, $F_{c}$, shown in Fig. 5 .

(2) Brittle Fracture of Plasticized High Polymers. High polymers such as polystyrene and cellulose acetate plasticized by dimethyl phthalate (DMP) were examined with the same apparatus mentioned above. In these case, brittle fracture occurred also after a large elastic elongation at a certain critical velocity of extension (cf. Fig. 1. b.). While the brittle velocity increased exponentially with increasing temperature as shown by the relation (1), the elongation at the brittle fracture remained almost constant. Thus these materials seemed to become brittle after elastic elongation which comes from the rubber-like elasticity and is characteristic of high polymers. Below the critical velocity, those high polymers showed remarkable spinning and did not break within the range of our experiment.

(3) Temperature Range for Creeping and Spinning. Spinnability of rosin at various concentrations was examined with the apparatus shown in Fig. 3 at various rates of pulling up and temperatures. It was confirmed that the specimen showed creeping or spinning within a certain range of temperature at a definite rate of pulling up. Below a lower critical temperature brittle fracture occurred and above a higher critical temperature spinning or dropping separation was observed. Thus two kinds of critical velocity $V_{n}$ and $V_{S}$ were obtained at various temperatures as shown in Fig. 6. Since $V_{n}$ and $V_{S}$ are given respectively by the exponential functions of the reciprocal of absolute temperature, the range of creeping or spinning may be given by the region between two straight lines, log

\footnotetext{
* Such behavior suggests that these materials have a nature of the Maxwell liquid.
} 
Brittle Fracture and Spinnability of Viscous Materials

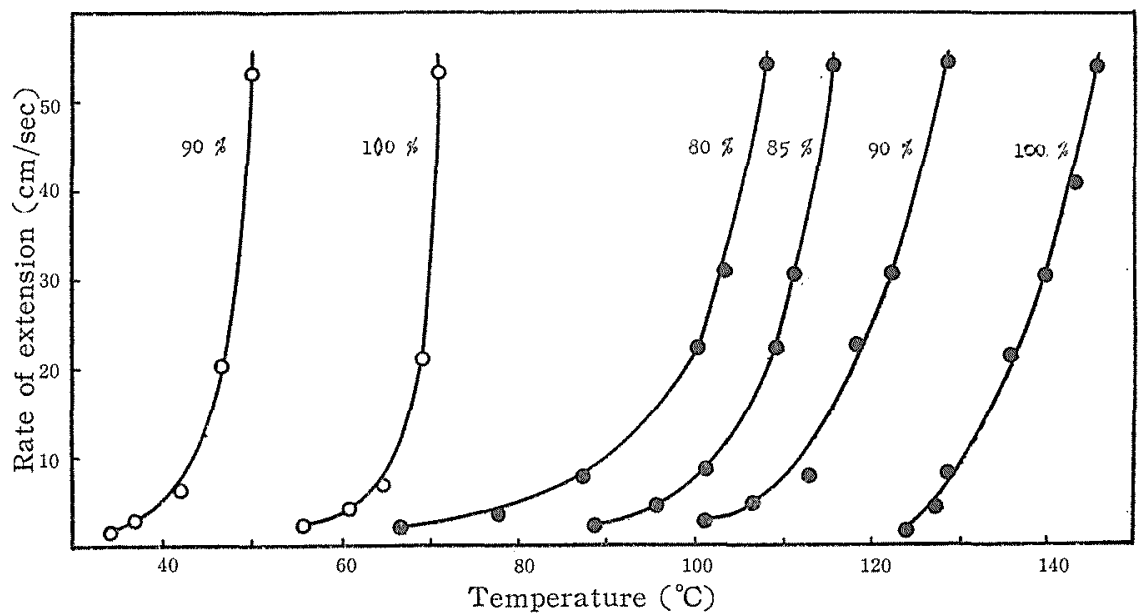

Fig. 6. Break-down limits of rosin-olive oil.

-O- brittle limit ; - dropping limit.

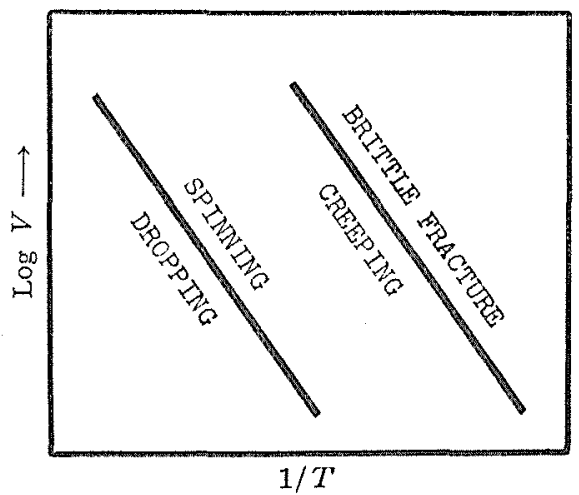

Fig. 7. Temperature range for creeping and spinning.

\section{$V_{B}-1 / T$ and $\log V_{S}-1 / T$, in Fig. 7.}

For asphalts, a similar relation was obtained. For plasticized high polymers such as cellulose acetate and polystyrene, the brittle condition could not be observed in this pulling up method, because cohesion of high polymers was superior to adhesion to the glass and the specimen detached from the glass rod at a criti-

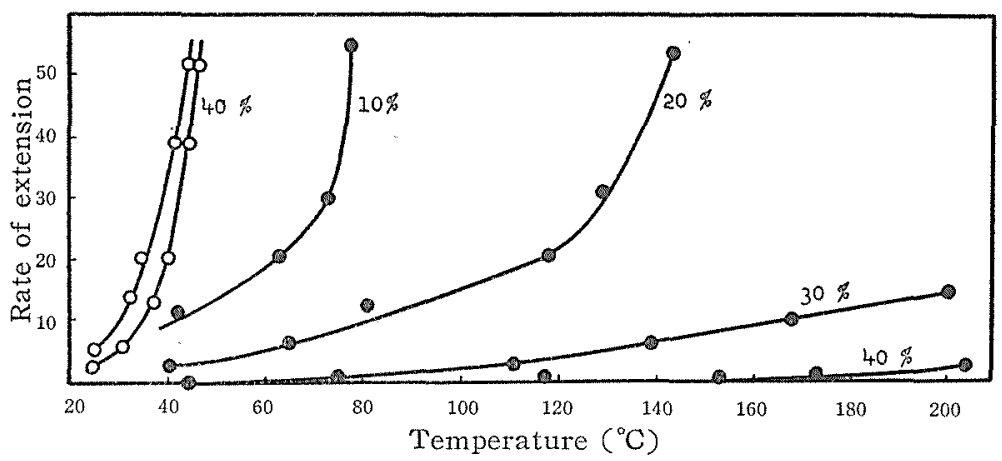

Fig. 8. Break-down limits of polystyrene-DOP.

-O- detaching limit; - - spinning limit. 


\section{Rempei GOTOH et al.}

cal rate of pulling. Fig. 8 shows the results obtained for the polystyrene-di-octyl phthalate.

(4) Surface Tension, Viscosity and Spinnability. Hirai, ${ }^{37}$ one of the present authors, intended to find rheologically some fundamental nature of spinning concerning relatively simple materials such as aqueous solutions of glycerine, cane sugar, glucose and glycols. It was shown that such liquids were very viscous at high concentrations and low temperatures, and became spinnable at certain rates of extension. Spinnability was observed by means of the pulling up method manually. At higher temperatures or at lower rate of extension, the liquids showed dropping separation. In the case of breaking of liquid thread, the two ends shrinked into drops as shown in Fig. 1 (d). Such character of spinning or breaking may be essentially the same with the dropping separation of rosin or asphalt which has been described in the previous section. From these facts, it may be suggested that spinning depends largely upon the viscosity and the surface tension as well as the rate of extension. In other words, in extension the surface tension leads the liquid thread to break, forming drops, but the viscosity keeps it from breaking.

Since the shrinking effect by the surface tension of liquids may be considered to be analogous to the effect of elasticity and given by $r / R_{0}$, the apparent relaxation time for shrinking, $\tau$, can be defined by the following relation,

$$
\tau=\frac{\gamma_{i}}{\gamma / R_{0}},
$$

where $r$ is the surface tension of the liquid and $R_{0}$ the radius of liquid thread.

Thus the spinning length of liquid $L$ may be expressed by the relation,

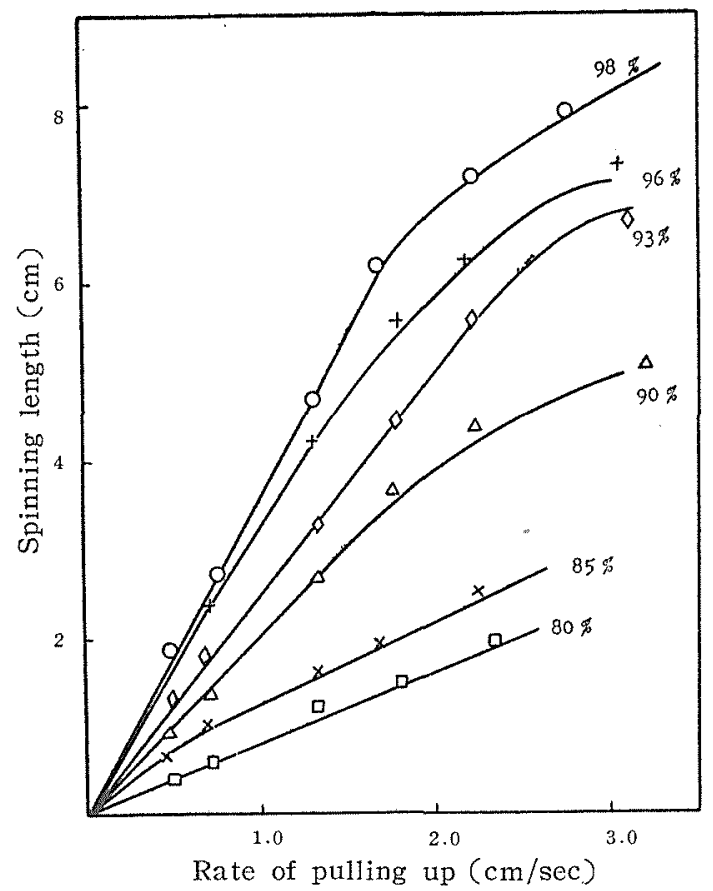

Fig. 9. Spinning length of glycerine (by Kawamura). 


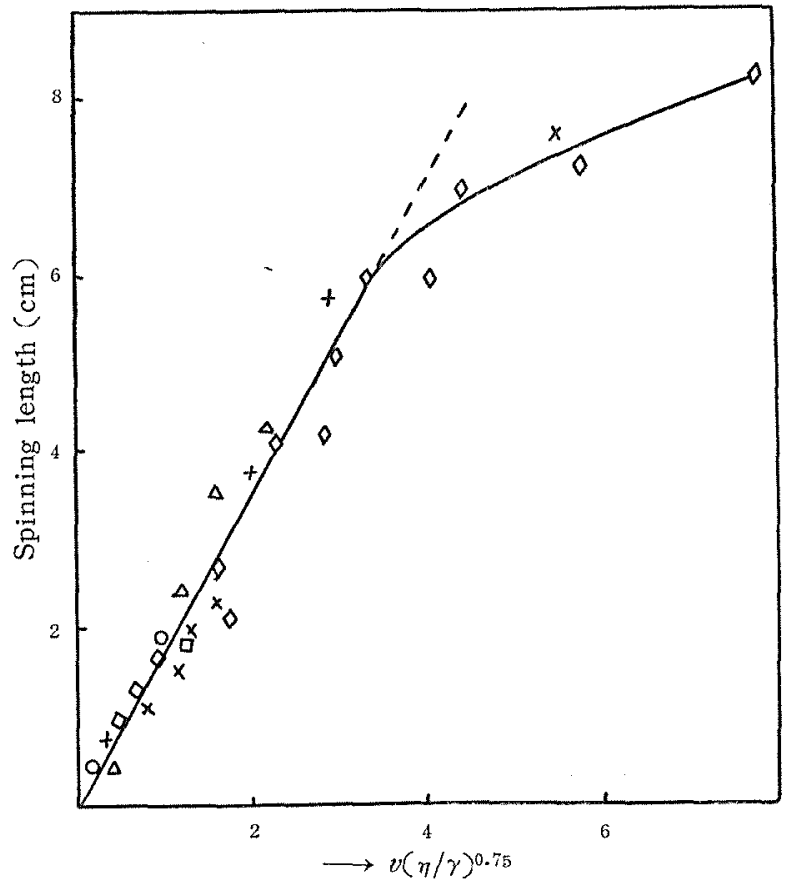

Fig. 10. Reduced curve for spinning length.

$$
L=v_{\tau}=v R_{0} \gamma_{/} / \gamma,
$$

where $v$ is the rate of extension. Fig. 9 shows the spinning length for the aqueous solution of glycerine at various concentrations which was reported by Kawamura. ${ }^{4}$ In Fig. 10, the spinning length $L$ was plotted against $v(\gamma / \gamma)^{0.75}$ and it was shown that the scattered points in Fig. 8 fell on a single line on the whole. This result shows that the relation (4) holds qualitatively, though some deviation from linear relation is seen in the region where $v$ or $x$ take higher values. Now, if the spinnability $S$ is defined by $L / R_{0}$, a dimensionless quantity, then

$$
S=\frac{L}{R_{0}}=v r_{1} / r \text {. }
$$

Assuming that the liquid is spinnable for $S \geq 1$, the spinning condition can be derived as follows:

$$
v \gamma / r \geq 1, \text { or } \eta \geq r / v \text {. }
$$

Plotting $\gamma$ and $r$ against the temperature respectively as shown in Fig. 11, the spinning temperature of the specimens at various concentrations can be determined by the cross points of the curves, $\gamma-T$ and $\gamma-T$; where the manual spinning velocity on an average is assumed to be $1 / 10 \mathrm{~cm}$ per second. In this case, the spinning condition can be given by the relation in c.g.s. unit,

$$
\gamma=\frac{1}{10} \gamma \text {. }
$$

As the surface tension of viscous materials could not be measured directly, it was estimated from the relation of Ramsay-Schield,

$$
\gamma=\left\{k /(M V)^{2 / 3}\right\}\left(T_{e}-T-6\right),
$$

or 
Rempei GOTOH et al.

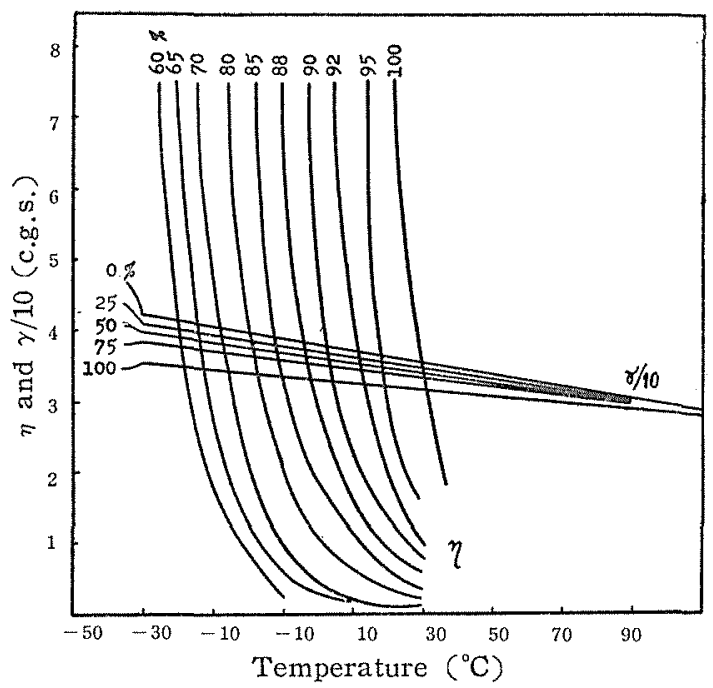

Fig. 11. Spinning temperature.

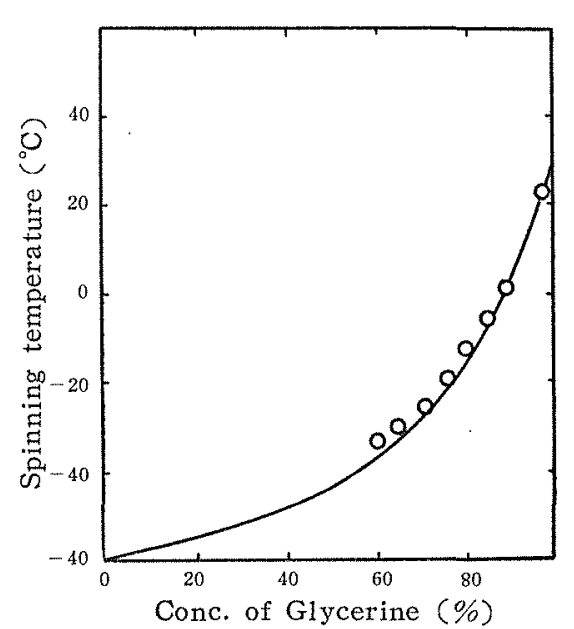

Fig. 12. Spinning temperature of glycerine.

$$
d r / d T=-k /(M V)^{2 / 3},
$$

where $k$ is a constant taking the values between 1 and $2, M$ the molecular weight, $V$ the molecular volume, $T_{u}$ the critical temperature and $T$ the absolute temperature.

In Fig. 11, the full line shows the spinning temperature at various concentrations of the aqueous solutions of glycerine which can be estimated by the relation (7) and Fig. 12, and points marked by hollow circles shows the actual spinning temperature obtained by the pulling up method. The critical temperature expected theoretically showed a good agreement with the experimental results. The aqueous solutions of cane sugar, glucose and propylene glycol showed similar behavior to the case mentioned above.

\section{CONCLUSION}

Rosin dissolved in olive oil and asphalt behave nearly as Newtonian liquids at a relatively low rate of strain, while they show brittle fracture or a solid nature above a critical rate of strain. Wheat-gluten, sealing wax, ebonite and pitch are rheologically analogous to the materials mentioned above. Further, the brittle strength of these materials takes values of the same order of magnitude with the viscous resistance at brittle break-down. Such behavior suggests that those materials have a nature of the Maxwell liquid.

At a higher temperature range, we can find a critical rate of strain for dropping break-down or spinning. Both the critical rate of strain for brittle fracture and for spinning are respectively expressed by exponential functions of the reciprocal of absolute temperature. Between these two limiting conditions, creeping or spinning can be seen.

The spinning condition of viscous liquids may be determined by the rate of strain and the apparent relaxation time which depends upon a balance between 
viscosity and surface tension.

The authors are grateful to the members of Central Technical Laboratory of the Nippon Oil Co. for the kind supply of asphalt samples. They are also indebted to the Ministry of Education of Japan for the research grant.

\section{REFERENCES}

(1) (a) A. A. Griffith, Phil. Trans. Roy. Soc., 221, 163 (1920); (b) M. Reiner, "Deformation and Flow," p. 295. H. K. Lewis \& Co. (1949) ; (c) A. Nadai, "Theory of Flow and Fractue of Solids," p 175. McGraw-Hill Book Co. (1950). ; (d) R. N. Haward, "The Strength of Plastics and Glass," p. 146. Cleaver-Hume Press Ltd. (1949); (e) J. C. Jager, "Elasticity, Fracture and Flow," p. 72. Methuen \& Co. (1956).

(2) (a) H. Erbring, Kolloid Beih. 44, 171 (1936); (b) T. Nakagawa, Bull. Chem. Soc. Japan, 25, 88 (1952) ; (c) H. Inagaki, J. Coll. Sci., 11, 226 (1956) ; (d) S. Shishido and Y. Ito, J. Chem, Soc. Japan, 78, 203, 608 (1957).

(3) N. Hirai, J. Chem. Soc. Japan, 75, 1021 (1954).

(4) N. Kawamura, J. Chem. Soc. Japan, 60, 88 (1939). 